\title{
On the distribution of primitive Pythagorean triangles
}

\author{
by \\ Kui LiU (Jinan)
}

1. Introduction and main results. A primitive Pythagorean triangle is a triple $(a, b, c)$ of natural numbers with $a^{2}+b^{2}=c^{2}, a<b, \operatorname{gcd}(a, b, c)$ $=1$. For a large real number $x$, let $P(x), A(x)$ and $H(x)$ denote the number of primitive Pythagorean triangles with perimeter, area and hypotenuse less than $x$, respectively. Many authors have studied the asymptotic behavior of $P(x), A(x)$ and $H(x)$.

In 1900, D. N. Lehmer [10] showed that

$$
P(x)=\frac{\log 2}{\pi^{2}} x+o(x), \quad H(x)=\frac{1}{2 \pi} x+o(x) .
$$

In 1948, D. H. Lehmer [9] proved

$$
P(x)=\frac{\log 2}{\pi^{2}} x+O\left(x^{1 / 2} \log x\right) .
$$

In 1955, J. Lambek and L. Moser [8] obtained (1.1) again and proved

$$
H(x)=\frac{1}{2 \pi} x+O\left(x^{1 / 2} \log x\right)
$$

and

$$
A(x)=c x^{1 / 2}+O\left(x^{1 / 3}\right),
$$

where $c=\left(2 \pi^{5}\right)^{-1 / 2} \Gamma^{2}(1 / 4)$.

In 1955, R. E. Wild [21] proved

$$
A(x)=c x^{1 / 2}-c^{\prime} x^{1 / 3}+R_{\text {area }}(x),
$$

where

$$
c^{\prime}=\frac{|\zeta(1 / 3)|\left(1+2^{-1 / 3}\right)}{\zeta(3 / 4)\left(1+4^{-1 / 3}\right)} \quad \text { and } \quad R_{\text {area }}(x)=O\left(x^{1 / 4} \log x\right)
$$

2010 Mathematics Subject Classification: Primary 11N37; Secondary 11L07.

Key words and phrases: primitive Pythagorean triangles, exponential sums, short interval. 
In 1980, J. Duttlinger and W. Schwarz [3] proved that there exists a positive constant $\delta>0$ such that

$$
R_{\text {area }}(x)=O\left(x^{1 / 4} e^{-\delta \log ^{1 / 2} x}\right) .
$$

It is difficult to reduce the exponents $1 / 2,1 / 2,1 / 4$ in the error terms of (1.1), (1.2), and (1.4), since the current technique depends on the strongest estimations of $\sum_{n \leq x} \mu(x)$, and the best zero-free regions of the Riemann zeta function so far. Therefore it is natural to search for stronger estimates under the Riemann Hypothesis $(\mathrm{RH})$. The exponent $1 / 4$ in $R_{\text {area }}(x)$ was improved by several authors under RH (see [11, [15], [14, [16], 22]). It is also of interest to consider the distributions of $P(x), A(x)$ and $H(x)$ unconditionally in short intervals.

In this paper, we shall prove the following

THEOREM 1.1. If $R H$ is true, then for any $\varepsilon>0$, we have

$$
P(x)=\frac{\log 2}{\pi^{2}} x+O\left(x^{\frac{5805}{15408}+\varepsilon}\right) .
$$

THEOREM 1.2. For any sufficiently small $\varepsilon>0$ and $x^{\frac{131}{416}+2 \varepsilon}<H \leq x$, we have

$$
P(x+H)-P(x)=\frac{\log 2}{\pi^{2}} H+O\left(H x^{-\varepsilon}\right) .
$$

TheOREM 1.3. For any sufficiently small $\varepsilon>0$ and $x^{\frac{435}{616}+2 \varepsilon}<H \leq x$, we have

$$
A(x+H)-A(x)=\frac{c}{2} H x^{-1 / 2}+O\left(H x^{-1 / 2-\varepsilon}\right),
$$

where $c$ is as in (1.3).

Notations. We use $\{t\}$ and $[t]$ to denote the fractional part and the integer part of $t$, respectively, and $\|t\|$ to denote the distance between $t$ and the nearest integer; $\varepsilon$ denotes a small positive constant which may be different at different occurrences; $\mu(n)$ denotes the Möbius function; $e(t)=$ $e^{2 \pi i t} ; m \sim M$ means $M<m \leq 2 M$ and $m \asymp M$ means $c_{1} M<m \leq c_{2} M$ for some $c_{2}>c_{1}>0$.

\section{Proof of Theorem 1.1}

2.1. Some preliminary lemmas. The following lemmas will be needed in our proof. Lemma 2.1 is the well-known Euler-Maclaurin summation formula (for example, see Theorem 2.1 of Chapter 2 in [17]). Lemma 2.2 is due to Vaaler [19]. Lemma 2.3 is Theorem 2.2 of Min [12] (see also Lemma 6 of Chapter 1 in [20]). Lemma 2.4 is Theorem 2 of Baker [1] with $(\kappa, \lambda)=$ 
$(1 / 2,1 / 2)$. Lemma 2.5 is Theorem 3 of Robert and Sargos 18 . Lemmas 2.62.8 are Lemma 6, Proposition 1 and Lemma 1 of Fouvry and Iwaniec [5], respectively.

Lemma 2.1. Suppose $f(u)$ is three times continuously differentiable on $[a, b]$. Then

$$
\begin{aligned}
\sum_{a<n \leq b} f(n)= & \int_{a}^{b} f(u) d u-f(b) \psi(b)+f(a) \psi(a)+\psi_{1}(b) f^{\prime}(b) \\
& -\psi_{1}(a) f^{\prime}(a)-\int_{a}^{b} \psi_{1}(u) f^{\prime \prime}(u) d u,
\end{aligned}
$$

where $\psi(t)=\{t\}-1 / 2, \psi_{1}(t)=\{t\}^{2} / 2-\{t\} / 2+1 / 12$.

Lemma 2.2. For any $H_{0} \geq 2$, we have

$$
\psi(t)=\sum_{1 \leq|h| \leq H_{0}} a(h) e(h t)+O\left(\sum_{0 \leq h \leq H_{0}} b(h) e(h t)\right),
$$

where $a(h) \ll 1 / h$ and $b(h) \ll 1 / H_{0}$.

Lemma 2.3. Let $A_{1}, \ldots, A_{5}$ be absolute positive constants. Suppose $f(x)$ and $g(x)$ are algebraic functions on $[a, b]$ and

$$
\begin{aligned}
& \frac{A_{1}}{R} \leq\left|f^{\prime \prime}(x)\right| \leq \frac{A_{2}}{R}, \quad\left|f^{\prime \prime \prime}(x)\right| \ll \frac{A_{3}}{R U}, \quad U>1, \\
& |g(x)| \leq A_{4} G, \quad\left|g^{\prime}(x)\right| \leq A_{5} G U_{1}^{-1}, \quad U_{1}>1 .
\end{aligned}
$$

Suppose $\alpha \leq f^{\prime}(x) \leq \beta$ for $x \in[a, b]$. Then

$$
\begin{aligned}
\sum_{a<n \leq b} g(n) e(f(n))= & e^{\pi i / 4} \sum_{\alpha \leq u \leq \beta} b_{u} \frac{g\left(n_{u}\right)}{\sqrt{f^{\prime \prime}\left(n_{u}\right)}} e\left(f\left(n_{u}\right)-u n_{u}\right) \\
& +O\left(G \log (\beta-\alpha+2)+G(b-a+R)\left(U^{-1}+U_{1}^{-1}\right)\right) \\
& +O\left(G \min \left[\sqrt{R}, \max \left(\frac{1}{\langle\alpha\rangle}, \frac{1}{\langle\beta\rangle}\right)\right]\right),
\end{aligned}
$$

where $n_{u}$ is the solution of $f^{\prime}(n)=u$,

$$
\begin{gathered}
\langle t\rangle= \begin{cases}\|t\| & \text { if } t \text { is not an integer, } \\
\beta-\alpha & \text { if } t \text { is an integer, }\end{cases} \\
b_{u}= \begin{cases}1 & \text { if } \alpha<u<\beta, \text { or both } \alpha \text { and } \beta \text { are not integers, } \\
1 / 2 & \text { if } u=\alpha \text { or } \beta \text { is an integer, }\end{cases}
\end{gathered}
$$

$$
\sqrt{f^{\prime \prime}}= \begin{cases}\sqrt{f^{\prime \prime}} & \text { if } f^{\prime \prime}>0, \\ i \sqrt{\left|f^{\prime \prime}\right|} & \text { if } f^{\prime \prime}<0 .\end{cases}
$$

LEMmA 2.4. Let $\alpha, \alpha_{1}, \alpha_{2}, z$ be real numbers such that $z \alpha \alpha_{1} \alpha_{2} \neq 0$ and $\alpha \notin \mathbb{N}$. Let $M \geq 2, M_{1} \geq 1, M_{2} \geq 1$, and let $a_{m}$ and $b_{m_{1} m_{2}}$ be complex 
numbers with $\left|a_{m}\right| \leq 1,\left|b_{m_{1} m_{2}}\right| \leq 1$. Let $F_{1}=|z| M^{\alpha} M_{1}^{\alpha_{1}} M_{2}^{\alpha_{2}}$. If $F_{1} \geq$ $M_{1} M_{2}$, then

$$
\begin{aligned}
& \sum_{m \sim M} \sum_{m_{1} \sim M_{1}} \sum_{m_{2} \sim M_{2}} a_{m} b_{m_{1} m_{2}} e\left(z m^{\alpha} m_{1}^{\alpha_{1}} m_{2}^{\alpha_{2}}\right) \\
& \quad \ll M M_{1} M_{2}\left\{\left(M_{1} M_{2}\right)^{-1 / 2}+F_{1}^{1 / 6} M^{-1 / 3}\left(M_{1} M_{2}\right)^{-1 / 6}\right\} \log \left(2 M M_{1} M_{2}\right) .
\end{aligned}
$$

Lemma 2.5. Let $\alpha(\alpha-1) \beta \gamma \neq 0, X>1$. Define

$$
\left|\sum_{M<m \leq 2 M} z_{m}\right|^{*}:=\max _{M<M_{1} \leq M_{2} \leq 2 M}\left|\sum_{m=M_{1}}^{M_{2}} z_{m}\right|
$$

and

$$
S:=\sum_{h \sim H} \sum_{n \sim N}\left|\sum_{M<m \leq 2 M} e\left(X \frac{m^{\alpha} h^{\beta} n^{\gamma}}{M^{\alpha} H^{\beta} N^{\gamma}}\right)\right|^{*},
$$

where $H, M, N$ are positive integers. Then for any $\varepsilon>0$, we have

$$
S \ll_{\varepsilon}(H N M)^{1+\varepsilon}\left(\left(\frac{X}{H N M^{2}}\right)^{1 / 4}+\frac{1}{M^{1 / 2}}+\frac{1}{X}\right) .
$$

Lemma 2.6. Let $0<L \leq N<v N<\lambda L$ and let $a_{l}$ be complex numbers with $\left|a_{l}\right| \leq 1$. Then

$$
\sum_{N<n<v N} a_{n}=\frac{1}{2 \pi} \int_{-L}^{L}\left(\sum_{L<l<\lambda L} a_{l} l^{-i t}\right) N^{i t}\left(v^{i t}-1\right) t^{-1} d t+O(\log (2+L)),
$$

where the constant implied in $O$ depends on $\lambda$ only.

Lemma 2.7. Let $\mathcal{X}$ and $\mathcal{Y}$ be two finite sets of real numbers, $\mathcal{X} \subset$ $[-X, X], \mathcal{Y} \subset[-Y, Y]$. Then for any complex functions $u(x)$ and $v(y)$,

$$
\begin{aligned}
&\left|\sum_{x \in \mathcal{X}} \sum_{y \in \mathcal{Y}} u(x) v(y) e(x y)\right|^{2} \\
& \leq 20(1+X Y) \sum_{\substack{x, x^{\prime} \in \mathcal{X} \\
\left|x-x^{\prime}\right| \leq Y^{-1}}}\left|u(x) u\left(x^{\prime}\right)\right| \sum_{\substack{y, y^{\prime} \in \mathcal{Y} \\
\left|y-y^{\prime}\right| \leq X^{-1}}}\left|v(y) v\left(y^{\prime}\right)\right| .
\end{aligned}
$$

Lemma 2.8. Let $\alpha \beta \neq 0, \Delta>0, M \geq 1$ and $N \geq 1$. Let $\mathcal{A}(M, N ; \Delta)$ be the number of quadruples $(m, \tilde{m}, n, \tilde{n})$ such that

$$
\left|\left(\frac{\tilde{m}}{m}\right)^{\alpha}-\left(\frac{\tilde{n}}{n}\right)^{\beta}\right|<\Delta
$$

with $M<m, \tilde{m} \leq 2 M$ and $N<n, \tilde{n} \leq 2 N$. Then

$$
\mathcal{A}(M, N ; \Delta) \ll M N \log (2 M N)+\Delta M^{2} N^{2} .
$$


2.2. Reduction of the problem. We always assume $x>0$ is a large real number in this paper. Define

$$
D_{\text {per }}(x):=\left\{(\alpha, \beta) \in \mathbb{R}^{2}: 2 \alpha^{2}+2 \alpha \beta \leq x, 0<\beta<\alpha\right\} .
$$

Let $L_{\text {per }}(x)$ and $L_{\text {per }}^{\prime}(x)$ denote the numbers of lattice points and of primitive lattice points inside $D_{\text {per }}(x)$, respectively ("per" comes from "perimeter").

LEMMA 2.9. We have

$$
P(x)=\sum_{k=0}^{\infty}(-1)^{k} L_{\text {per }}^{\prime}\left(\frac{x}{2^{k}}\right), \quad L_{\text {per }}^{\prime}(x)=\sum_{m=1}^{\infty} \mu(m) L_{\text {per }}\left(\frac{x}{m^{2}}\right) .
$$

Proof. This is contained in Lambek and Moser [8].

For $L_{\text {per }}(x)$, we have the following

LEMma 2.10. We have

$$
L_{\mathrm{per}}(x)=c_{1} x-c_{2} x^{1 / 2}+E_{\mathrm{per}}(x),
$$

where

$$
\begin{aligned}
c_{1} & =\frac{\log 2}{4}, \quad c_{2}=\frac{\sqrt{2}+1}{4}, \\
E_{\mathrm{per}}(x) & =-\sum_{\frac{1}{2} x^{1 / 2}<d \leq \frac{1}{\sqrt{2}} x^{1 / 2}} \psi\left(\frac{x}{2 d}\right)+O(1) .
\end{aligned}
$$

Proof. Let $x^{\prime}=\frac{1}{2}\left((2 x+1)^{1 / 2}-1\right)$. By the definition of $L_{\text {per }}(x)$, we have

$$
\begin{aligned}
L_{\mathrm{per}}(x) & =\sum_{\substack{2 d^{2}+2 d l \leq x \\
l<d}} 1=\sum_{\substack{2 d^{2}+2 d l \leq x \\
l \leq d}} 1-\sum_{\substack{2 d^{2}+2 d l \leq x \\
d=l}} 1 \\
& =\sum_{d \leq x^{\prime} l \leq \min \left(d, \frac{x}{2 d}-d\right)} 1-\left[\frac{x^{1 / 2}}{2}\right] \\
& =\sum_{d \leq \frac{1}{2} x^{1 / 2}} d+\sum_{\frac{1}{2} x^{1 / 2}<d \leq x^{\prime}}\left(\left[\frac{x}{2 d}\right]-d\right)-\left[\frac{x^{1 / 2}}{2}\right] \\
& =\Sigma_{1}+2 \Sigma_{2}-\Sigma_{3}-x^{1 / 2} / 2+O(1),
\end{aligned}
$$

where

$$
\Sigma_{1}=\sum_{\frac{1}{2} x^{1 / 2}<d \leq x^{\prime}}\left[\frac{x}{2 d}\right], \quad \Sigma_{2}=\sum_{d \leq \frac{1}{2} x^{1 / 2}} d, \quad \Sigma_{3}=\sum_{d \leq x^{\prime}} d .
$$

It is easy to see that

$$
\Sigma_{2}=\frac{1}{2}\left[\frac{x^{1 / 2}}{2}\right]\left(\left[\frac{x^{1 / 2}}{2}\right]+1\right)=\frac{x}{8}-\frac{x^{1 / 2}}{2} \psi\left(\frac{x^{1 / 2}}{2}\right)+O(1) .
$$


Similarly,

$$
\Sigma_{3}=\frac{x}{4}-\frac{\sqrt{2}}{4} x^{1 / 2}-\frac{\sqrt{2}}{2} x^{1 / 2} \psi\left(x^{\prime}\right)+O(1) .
$$

For $\Sigma_{1}$, we have

$$
\begin{aligned}
\Sigma_{1} & =\sum_{\frac{1}{2} x^{1 / 2}<d \leq x^{\prime}}\left(\frac{x}{2 d}-\psi\left(\frac{x}{2 d}\right)-\frac{1}{2}\right) \\
& =\frac{x}{2} \sum_{\frac{1}{2} x^{1 / 2}<d \leq x^{\prime}} \frac{1}{d}-\sum_{\frac{1}{2} x^{1 / 2}<d \leq x^{\prime}} \psi\left(\frac{x}{2 d}\right)-\frac{1}{2} \sum_{\frac{1}{2} x^{1 / 2}<d \leq x^{\prime}} 1 \\
& =\frac{x}{2} \sum_{\frac{1}{2} x^{1 / 2}<d \leq x^{\prime}} \frac{1}{d}-\frac{\sqrt{2}-1}{4} x^{1 / 2}-\sum_{\frac{1}{2} x^{1 / 2}<d \leq \frac{\sqrt{2}}{2} x^{1 / 2}} \psi\left(\frac{x}{2 d}\right)+O(1) .
\end{aligned}
$$

By Lemma 2.1 we have

$$
\begin{aligned}
& \sum_{\frac{1}{2} x^{1 / 2}<d \leq x^{\prime}} \frac{1}{d} \\
= & \int_{\frac{1}{2} x^{1 / 2}}^{x^{\prime}} \frac{1}{t} d t-\sqrt{2} x^{-1 / 2} \psi\left(x^{\prime}\right)+2 x^{-1 / 2} \psi\left(\frac{x^{1 / 2}}{2}\right)+O\left(\frac{1}{x}\right) \\
= & \log \left(\frac{1}{2}\left((2 x+1)^{1 / 2}-1\right)\right)-\log \left(\frac{x^{1 / 2}}{2}\right)-\sqrt{2} x^{-1 / 2} \psi\left(x^{\prime}\right) \\
& +2 x^{-1 / 2} \psi\left(\frac{x^{1 / 2}}{2}\right)+O\left(\frac{1}{x}\right) \\
= & \frac{\log 2}{2}-\frac{\sqrt{2}}{2} x^{-1 / 2}-\sqrt{2} x^{-1 / 2} \psi\left(x^{\prime}\right)+2 x^{-1 / 2} \psi\left(\frac{x^{1 / 2}}{2}\right)+O\left(\frac{1}{x}\right) .
\end{aligned}
$$

Now Lemma 2.10 follows by (2.4)-(2.8).

Lemma 2.10 is important in our proofs of Theorems 1.1 and 1.2. Note that the expression (2.3) of $E_{\text {per }}(x)$ is similar to

$$
\Delta(x)=-2 \sum_{d \leq x^{1 / 2}} \psi\left(\frac{x}{d}\right)+O(1),
$$

which appears as the error term in the Dirichlet divisor problem. Therefore, many approaches used in the study of $\Delta(x)$ can also be applied to the estimate of $E_{\text {per }}(x)$. The latest result for the upper bound of $\Delta(x)$ reads (see Huxley [6])

$$
\Delta(x) \ll x^{\frac{131}{416}}(\log x)^{\frac{26947}{8320}} .
$$


Similarly, combining (2.3) with the results of Huxley [6], we immediately get

$$
E_{\text {per }}(x) \ll x^{\frac{131}{416}+\varepsilon} \text {. }
$$

Suppose $1 \leq y \ll x^{1 / 2}$ is a parameter to be determined. By Lemma 2.10, we decompose $L_{\text {per }}^{\prime}(x)$ as

$$
\begin{aligned}
L_{\mathrm{per}}^{\prime}(x) & =\sum_{m \leq y} \mu(m) L_{\mathrm{per}}\left(\frac{x}{m^{2}}\right)+\sum_{m>y} \mu(m) L_{\mathrm{per}}\left(\frac{x}{m^{2}}\right) \\
& =c_{1} x \sum_{m \leq y} \frac{\mu(m)}{m^{2}}-c_{2} x^{1 / 2} \sum_{m \leq y} \frac{\mu(m)}{m}+S_{1}+S_{2},
\end{aligned}
$$

where $c_{1}, c_{2}$ are defined by $(2.2)$, and

$$
\begin{aligned}
& S_{1}=\sum_{m \leq y} \mu(m) E_{\mathrm{per}}\left(\frac{x}{m^{2}}\right), \\
& S_{2}=\sum_{m>y} \mu(m) L_{\mathrm{per}}\left(\frac{x}{m^{2}}\right) .
\end{aligned}
$$

2.3. Estimation of $S_{2}$. We shall estimate $S_{2}$ in a standard way. The key step is a familiar contour integration technique in the spirit of Montgomery and Vaughan [13, which is used in many occasions when estimating primitive lattice points (for example, see [7], [16], 23]). We only give an outline of the technique here.

Suppose $s=\sigma+i t$. For $\sigma>1$, define

$$
Z(s):=\sum_{n=1}^{\infty} \frac{r(n)}{n^{s}}, \quad \text { where } \quad r(n):=\sum_{\substack{2 d^{2}+2 d l=n \\ l<d}} 1 .
$$

Obviously, $r(n) \leq d(n) \ll n^{\varepsilon}$.

LEMMA 2.11. $Z(s)$ has the following properties:

(i) $Z(s)$ has an analytic continuation to $\sigma>1 / 4$, which has two simple poles at $s=1,1 / 2$ with residues $c_{1},-c_{2} / 2$ respectively, where $c_{1}, c_{2}$ are defined by $(2.2)$.

(ii) Suppose $1 / 4<\theta<1 / 2$ is the smallest $\alpha$ such that $E_{\mathrm{per}}(x) \ll x^{\alpha}$. For any real parameter $T \geq 10$, we have

$$
\int_{T}^{2 T}\left|Z\left(\frac{9+4 \theta}{16}+i t\right)\right|^{2} d t \ll T^{1+\varepsilon} .
$$

Proof. This can be proved in the same way as Lemma 5.2 of Zhai [22] (quoted as Lemma 4.4 in this paper) with slight modifications only.

To estimate $S_{2}$, we need the following lemma, the proof of which is contained in Nowak [16]. 
Lemma 2.12. Assume RH. Suppose that for some $\sigma \geq 1 / 2, T \geq 10$,

$$
\int_{T}^{2 T}|Z(\sigma+i t)|^{2} d t \ll T^{1+\varepsilon} .
$$

Then

$$
S_{2}=c_{1} x \sum_{m>y} \frac{\mu(m)}{m^{2}}+O\left(x^{\theta+\varepsilon}+x^{\sigma+\varepsilon} y^{1 / 2-2 \sigma}\right),
$$

where $\theta$ is as in Lemma 2.11(ii).

By (2.9) and Lemmas 2.11, 2.12, we take $\theta=\frac{131}{416}+\varepsilon, \sigma=\frac{9+4 \theta}{16}$ and $y=x^{\frac{651}{1926}}$, thus

$$
\begin{aligned}
S_{2} & =c_{1} x \sum_{m>y} \frac{\mu(m)}{m^{2}}+O\left(x^{\theta+\varepsilon}+x^{(9+4 \theta) / 16+\varepsilon} y^{-(5+4 \theta) / 8}\right) \\
& =c_{1} x \sum_{m>y} \frac{\mu(m)}{m^{2}}+O\left(x^{\frac{5805}{15408}+\varepsilon}\right) .
\end{aligned}
$$

2.4. Estimation of $S_{1}$. By (2.11) and Lemma 2.10,

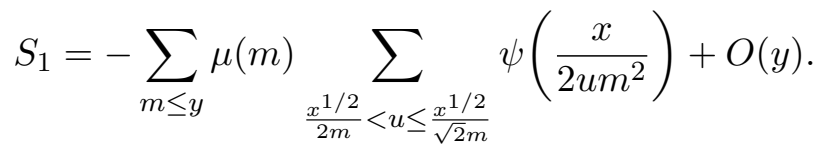

Our aim is to prove $S_{1} \ll x^{\frac{5805}{15408}+\varepsilon}$ for $y=x^{\frac{651}{1926}}$. By Lemma 2.2, we have

$$
S_{1} \ll\left|S_{1}\left(x, y, H_{0}\right)\right|+\left|S_{2}\left(x, y, H_{0}\right)\right|+x^{1 / 2+\varepsilon} H_{0}^{-1}+y,
$$

where

$$
\begin{aligned}
& S_{1}\left(x, y, H_{0}\right)=\sum_{m \leq y} \mu(m) \sum_{h \leq H_{0}} a(h) \sum_{\frac{x^{1 / 2}}{2 m}<u \leq \frac{x^{1 / 2}}{\sqrt{2} m}} e\left(\frac{h x}{2 u m^{2}}\right), \\
& S_{2}\left(x, y, H_{0}\right)=\sum_{m \leq y}\left|\sum_{h \leq H_{0}} b(h) \sum_{\frac{x^{1 / 2}}{2 m}<u \leq \frac{x^{1 / 2}}{\sqrt{2} m}} e\left(\frac{h x}{2 u m^{2}}\right)\right|,
\end{aligned}
$$

with $a(h) \ll 1 / h$ and $b(h) \ll 1 / H_{0}$. From now on we will take $H_{0}=x^{\frac{1899}{15408}}$, thus

$$
S_{1} \ll\left|S_{1}\left(x, y, H_{0}\right)\right|+\left|S_{2}\left(x, y, H_{0}\right)\right|+x^{\frac{5805}{15008}+\varepsilon} .
$$


We shall only prove $S_{1}\left(x, y, H_{0}\right) \ll x^{\frac{5805}{15408}+\varepsilon}$. The proof of $S_{2}\left(x, y, H_{0}\right) \ll$ $x^{\frac{5805}{15408}+\varepsilon}$ is similar and easier. By Lemma 2.3,

$$
\begin{aligned}
& S_{1}\left(x, y, H_{0}\right) \\
& \ll x^{1 / 4}\left|\sum_{m \leq y} \mu(m) \sum_{h \leq H_{0}} h a(h) \sum_{h \leq v \leq 2 h} \frac{1}{m^{1 / 2} h^{3 / 4} v^{3 / 4}} e\left(\frac{\sqrt{2} x^{1 / 2} h^{1 / 2} v^{1 / 2}}{m}\right)\right| \\
& \quad+x^{\frac{5805}{15408}} .
\end{aligned}
$$

Let $n=h v$. By a splitting argument and partial summation, we have

$$
x^{-\varepsilon} S_{1}\left(x, y, H_{0}\right) \ll x^{1 / 4} \sup _{\substack{1 \ll M \ll y \\ 1 \ll N \ll H_{0}^{2}}} M^{-1 / 2} N^{-3 / 4}\left|S_{1}^{*}(x, M, N)\right|+x^{\frac{5805}{15408}}
$$

where

$$
S_{1}^{*}(x, M, N)=\sum_{m \sim M} \mu(m) \sum_{n \sim N} a_{n} e\left(\frac{\sqrt{2} x^{1 / 2} n^{1 / 2}}{m}\right)
$$

with $\left|a_{n}\right| \leq 1$.

We shall estimate $M^{-1 / 2} N^{-3 / 4} S_{1}^{*}(x, M, N)$ in three cases.

CASE 1: $M \leq x^{3 / 11}, N \ll H_{0}^{2}$. Note that $S_{1}^{*}(x, M, N)$ is the same exponential sum as in [2] apart from the constant $\sqrt{2}$. We use the result of [2] directly to obtain

$$
x^{-\varepsilon} M^{-1 / 2} N^{-3 / 4}\left|S_{1}^{*}(x, M, N)\right| \ll x^{5 / 44},
$$

for $M \ll x^{3 / 11}$ and $N \ll x^{3 / 11}$. This estimate is acceptable for us, since $H_{0}^{2} \ll x^{3 / 11}$ and $\frac{1}{4}+\frac{5}{44}<\frac{5805}{15408}$.

CASE 2: $x^{3 / 11}<M \ll y, x^{-\frac{1953}{3852}} M^{2} \leq N \ll H_{0}^{2}$. By Lemma 2.4 (take $\left.m_{1}=1, m_{2}=n\right)$,

$$
x^{-\varepsilon} M^{-1 / 2} N^{-3 / 4}\left|S_{1}^{*}(x, M, N)\right| \ll M^{1 / 2} N^{-1 / 4}+x^{1 / 12} N^{1 / 6} \ll x^{\frac{1953}{15408}} .
$$

CASE $3: x^{3 / 11}<M \ll y, N \ll x^{-\frac{1953}{3852}} M^{2}$. By the skillful decomposition due to Montgomery and Vaughan [13] and a splitting argument, we can decompose $S_{1}^{*}(x, M, N)$ into at most $O(\log M)$ sums of the following two forms:

$$
\begin{array}{ll}
\Sigma_{1}=\sum_{n \sim N} a_{n} \sum_{k \sim K} b_{k} \sum_{r \sim M k^{-1}} e\left(\frac{\sqrt{2} x^{1 / 2} n^{1 / 2}}{k r}\right), & K \ll M^{1 / 3}, \\
\Sigma_{2}=\sum_{n \sim N} a_{n} \sum_{k \sim K} b_{k} \sum_{r \sim M k^{-1}} c_{r} e\left(\frac{\sqrt{2} x^{1 / 2} n^{1 / 2}}{k r}\right), & M^{1 / 3} \ll K \ll M^{1 / 2},
\end{array}
$$


where $b_{k} \ll M^{\varepsilon}$ and $c_{r} \ll M^{\varepsilon}$. Applying Lemma 2.5 to $\Sigma_{1}$ with $X, M, H$, $N$ replaced by $x^{1 / 2} M^{-1} N^{1 / 2}, M K^{-1}, K, N$, respectively, we get

$$
\begin{aligned}
x^{-\varepsilon} M^{-1 / 2} & N^{-3 / 4} \Sigma_{1} \\
& \ll x^{1 / 8} M^{-1 / 4} N^{1 / 8} K^{1 / 4}+N^{1 / 4} K^{1 / 2}+x^{-1 / 2} M^{3 / 2} N^{-1 / 4} \\
& \ll x^{\frac{1899}{30816}} M^{1 / 12}+x^{-\frac{1953}{15408}} M^{2 / 3}+x^{-1 / 2} M^{3 / 2} \ll x^{\frac{1953}{15408}} .
\end{aligned}
$$

Applying Lemma 2.6 to the sum over $r$ in $\Sigma_{2}$, we get

$$
\begin{aligned}
\Sigma_{2} \ll & \int_{-\frac{1}{2} M K^{-1}}^{\frac{1}{2} M K^{-1}} \mid t^{-1}\left(2^{i t}-1\right) \sum_{n \sim N} a_{n} \sum_{k \sim K} b_{k}\left(M k^{-1}\right)^{i t} \\
& \times \sum_{\frac{1}{2} M k^{-1}<r<2 M k^{-1}} c_{r} r^{i t} e\left(\frac{\sqrt{2} x^{1 / 2} n^{1 / 2}}{k r}\right) \mid d t+O(N K \log x) .
\end{aligned}
$$

Applying Lemma 2.7 to the three-dimensional exponential sum in the integral with $\mathcal{X}=\sqrt{2} x^{1 / 2} r^{-1}$ and $\mathcal{Y}=n^{1 / 2} k^{-1}$, we get

$$
x^{-\varepsilon} \Sigma_{2} \ll x^{1 / 4} N^{1 / 4} M^{-1 / 2} \mathcal{A}_{1}^{1 / 2} \mathcal{A}_{2}^{1 / 2}+N K,
$$

where

$$
\mathcal{A}_{1}=\sum_{\substack{\left|x^{1 / 2} r_{1}^{-1}-x^{1 / 2} r_{2}^{-1}\right| \ll N^{-1 / 2} K \\ r_{1}, r_{2} \sim M K^{-1}}} 1, \quad \mathcal{A}_{2}=\sum_{\substack{\left|n_{1}^{1 / 2} k_{1}^{-1}-n_{2}^{1 / 2} k_{2}^{-1}\right| \ll x^{-1 / 2} M K^{-1} \\ r_{1}, r_{2} \sim M K^{-1} \\ k_{1}, k_{2} \sim K}} 1 .
$$

For $\mathcal{A}_{1}$ we have

$$
\begin{aligned}
\mathcal{A}_{1} & \ll \sum_{\substack{\left|r_{1}-r_{2}\right| \ll x^{-1 / 2} M^{2} N^{-1 / 2} K^{-1} \\
r_{1}, r_{2} \sim M K^{-1}}} 1 \\
& \ll M K^{-1}\left(1+x^{-1 / 2} M^{2} N^{-1 / 2} K^{-1}\right) .
\end{aligned}
$$

Applying Lemma 2.8 to $\mathcal{A}_{2}$, we have

$$
\mathcal{A}_{2} \ll N K \log (N K)+\left(x^{-1 / 2} M N^{-1 / 2}\right) N^{2} K^{2} .
$$

Combining (2.19), (2.20) with (2.18) yields

$$
\begin{aligned}
x^{-\varepsilon} M^{-1 / 2} N^{-3 / 4} \Sigma_{2} & \\
& \ll x^{-1 / 4} M+N^{1 / 4} K^{1 / 2}+M^{1 / 2} N^{-1 / 4} K^{-1 / 2}+x^{1 / 4} M^{-1 / 2} \\
& \ll x^{-1 / 4} M+x^{-\frac{1953}{15408}} M^{3 / 4}+M^{1 / 3}+x^{\frac{1}{4}} M^{-1 / 2} \ll x^{\frac{1953}{15408}} .
\end{aligned}
$$

Now $S_{1} \ll x^{\frac{5805}{15408}+\varepsilon}$ follows by combining the estimates in Cases $1-3$ with (2.14) and (2.15). 
2.5. Proof of Theorem 1.1. Under the assumption of RH, we have

$$
c_{3} x^{1 / 2} \sum_{m \leq y} \frac{\mu(m)}{m} \ll x^{1 / 2+\varepsilon} y^{-1 / 2} \ll x^{\frac{5805}{15408}} .
$$

Combining this and the estimates for $S_{1}, S_{2}$ with (2.10), we get

$$
L_{\text {per }}^{\prime}(x)=c_{1} \zeta(2)^{-1} x+O\left(x^{\frac{5805}{15408}+\varepsilon}\right) .
$$

Theorem 1.1 follows immediately from (2.21) and Lemma 2.9.

3. Proof of Theorem 1.2. By (1.1), it is easy to see that (1.7) holds for $x^{1 / 2+2 \varepsilon}<H \leq x$. Hence we only need to prove (1.7) for $x^{\frac{131}{416}+2 \varepsilon}<H \leq$ $x^{1 / 2+2 \varepsilon}$. By Lemma 2.9, we write

$$
\begin{aligned}
L_{\mathrm{per}}^{\prime}(x+H)-L_{\mathrm{per}}^{\prime}(x) & =\sum_{m=1}^{\infty} \mu(m)\left(L_{\mathrm{per}}\left(\frac{x+H}{m^{2}}\right)-L_{\mathrm{per}}\left(\frac{x}{m^{2}}\right)\right) \\
& =\sum_{m \leq x^{\varepsilon}}+\sum_{m>x^{\varepsilon}},
\end{aligned}
$$

say. By Lemma 2.10 and (2.9), we have

$$
\sum_{m \leq x^{\varepsilon}}=c_{1} \zeta(2)^{-1} H+O\left(x^{\frac{131}{416}+\varepsilon}\right) .
$$

To estimate $\sum_{m>x^{\varepsilon}}$, we need the following lemma which is contained in the proof of Theorem 1 of Filaseta and Trifonov [4].

LEMma 3.1. For any integer $k \geq 1$, we have

$$
\sum_{\substack{x \leq n m^{k}<x+y \\ m>x^{\varepsilon}}} 1 \ll y x^{-\varepsilon / 2}+x^{1 /(2 k+1)+\varepsilon} .
$$

Now we estimate $\sum_{m>x^{\varepsilon}}$. Note that $L_{\text {per }}(x)=\sum_{n \leq x} r(n)$. Hence by Lemma 3.1,

$$
\sum_{m>x^{\varepsilon}}=\sum_{\substack{x \leq n m^{2}<x+H \\ m>x^{\varepsilon}}} r(n) \mu(m) \ll x^{\varepsilon^{2}} \sum_{\substack{x \leq n m^{2}<x+H \\ m>x^{\varepsilon}}} 1 \ll H x^{-\varepsilon}+x^{1 / 5+3 \varepsilon},
$$

where we have used the estimate $r(n) \ll n^{\varepsilon}$. By the above arguments, we get

$$
L_{\text {per }}^{\prime}(x+H)-L_{\text {per }}^{\prime}(x)=c_{1} \zeta(2)^{-1} H+O\left(H x^{-\varepsilon}+x^{\frac{131}{416}+\varepsilon}\right) .
$$

This together with Lemma 2.9 yields Theorem 1.2.

\section{Proof of Theorem 1.3. Define}

$$
D_{\text {area }}(x):=\left\{(\alpha, \beta) \in \mathbb{R}^{2}: \alpha \beta\left(\alpha^{2}-\beta^{2}\right)<x, 0<\beta<\alpha\right\} \text {. }
$$


Let $L_{\text {area }}(x)$ and $L_{\text {area }}^{\prime}(x)$ denote the number of lattice points and primitive lattice points inside $D_{\text {area }}(x)$, respectively. For $s=\sigma+i t, \sigma>1$, define

$$
f_{1}(k ; y):=\sum_{\substack{d^{4} m n\left(m^{2}-n^{2}\right)=k \\ d>y}} \mu(d), \quad f_{2}(k):=\sum_{m n\left(m^{2}-n^{2}\right)=k} 1,
$$

and

$$
F_{1}(s ; y):=\sum_{k=1}^{\infty} \frac{f_{1}(k ; y)}{k^{s}}, \quad F_{2}(s):=\sum_{k=1}^{\infty} \frac{f_{2}(k)}{k^{s}}, \quad F(s ; y):=\sum_{d>y} \frac{\mu(d)}{d^{4 s}} .
$$

Obviously

$$
F_{1}(s ; y)=F_{2}(s) F(s ; y) .
$$

To prove Theorem 1.3 we need the following lemmas. Lemma 4.1 is due to Lambek and Moser [8]. Lemma 4.2 is due to Nowak [16, p. 176]. Lemmas 4.3 and 4.4 are (4.7) and Lemma 5.2 of Zhai [22], respectively. Lemma 4.5 is a well-known result on the mean value of Dirichlet polynomial (for example, see Theorem 2.2 of Chapter 29 in [17] with $q=1$ ).

LEMMA 4.1. We have

$$
A(x)=\sum_{k=0}^{\infty}(-1)^{k} L_{\text {area }}^{\prime}\left(\frac{x}{4^{k}}\right), \quad \text { where } \quad L_{\text {area }}^{\prime}(x)=\sum_{m=1}^{\infty} \mu(m) L_{\text {area }}\left(\frac{x}{m^{4}}\right) .
$$

LEMmA 4.2. We have

$$
L_{\text {area }}(x)=c_{3} x^{1 / 2}-c_{4} x^{1 / 3}+F_{\text {area }}(x),
$$

where

$$
\begin{gathered}
c_{3}=\frac{\Gamma^{2}(1 / 4)}{4(2 \pi)^{1 / 2}}, \quad c_{4}=|\zeta(1 / 3)|\left(1+2^{-1 / 3}\right), \\
F_{\text {area }}(x) \ll x^{\frac{23}{146}}(\log x)^{\frac{315}{146}} .
\end{gathered}
$$

LEMMA 4.3. For $1 \leq y \ll x^{1 / 4}$, we have

$$
\sum_{d \leq y}\left|F_{\text {area }}\left(\frac{x}{d^{4}}\right)\right| \ll x^{\frac{127}{616}} \log ^{\frac{963}{308}} x+x^{1 / 8} y^{1 / 2} \log ^{4} x .
$$

Lemma 4.4. $F_{2}(s)$ has the following properties:

(i) $F_{2}(s)$ has an analytic continuation to $\sigma>1 / 8$. It has two simple poles at $s=1 / 2,1 / 3$ with residues $c_{3} / 2,-c_{4} / 3$, respectively, where $c_{3}, c_{4}$ are defined in Lemma 4.2 .

(ii) $F_{2}(\sigma+i t) \ll \min \left(\log |t|, \frac{2}{2 \sigma-1}\right)$ for $\sigma \geq 1 / 2$ and $|t| \geq 2$.

(iii) $F_{2}(\sigma+i t) \ll|t|^{(4-8 \sigma) / 3} \log t$ uniformly for $1 / 8<\sigma_{1} \leq \sigma \leq 1 / 2$ and $|t| \geq 2$. 
(iv) For any $T \geq 10$, we have

$$
\int_{T}^{2 T}\left|F_{2}\left(\frac{24}{73}+i t\right)\right|^{2} d t \ll T \log ^{7} T .
$$

Lemma 4.5. For any arithmetic function $a: \mathbb{N} \rightarrow \mathbb{C}$, we have

$$
\int_{-T}^{T}\left|\sum_{n \leq x} \frac{a(n)}{n^{i t}}\right|^{2} d t \ll \sum_{n \leq x}(T+n)|a(n)|^{2} .
$$

From (1.4) and (1.5), it is easy to see that (1.8) holds for $x^{3 / 4+\varepsilon}<H \leq x$, hence we only need to prove (1.8) for $x^{\frac{435}{616}+2 \varepsilon}<H \leq x^{3 / 4+\varepsilon}$. Lemma 4.1 gives

$$
L_{\text {area }}^{\prime}(x+H)-L_{\text {area }}^{\prime}(x)=\sum_{d=1}^{\infty} \mu(d)\left(L_{\text {area }}\left(\frac{x+H}{d^{4}}\right)-L_{\text {area }}\left(\frac{x}{d^{4}}\right)\right) .
$$

Suppose $x^{\varepsilon}<y \ll x^{1 / 4}$ is a parameter to be determined. We divide the sum over $d$ into two sums,

$$
L_{\text {area }}^{\prime}(x+H)-L_{\text {area }}^{\prime}(x)=\sum_{d \leq y}+\sum_{d>y},
$$

say. By Lemmas 4.2 and 4.3 ,

$$
\begin{aligned}
\sum_{d \leq y}= & c_{3}\left((x+H)^{1 / 2}-x^{1 / 2}\right) \sum_{d \leq y} \frac{\mu(d)}{d^{2}} \\
& -c_{4}\left((x+H)^{1 / 3}-x^{1 / 3}\right) \sum_{d \leq y} \frac{\mu(d)}{d^{4 / 3}} \\
& +O\left(x^{\frac{127}{616}+\varepsilon}+x^{1 / 8+\varepsilon} y^{1 / 2}\right) .
\end{aligned}
$$

By the definition of $L_{\text {area }}(x)$, we have

$$
\begin{aligned}
& \sum_{d>y}=\sum_{d>y} \mu(d) \sum_{\frac{x}{d^{4}}<m n\left(m^{2}-n^{2}\right)<\frac{x+H}{d^{4}}} 1 \\
& =\sum_{\substack{x<d^{4} m n\left(m^{2}-n^{2}\right) \leq x+H \\
d>y}} \mu(d)=\sum_{x<k \leq x+H} f_{1}(k ; y) .
\end{aligned}
$$

By Perron's formula,

$$
\sum_{x<k \leq x+H} f_{1}(k ; y)=\frac{1}{2 \pi i} \int_{1+\varepsilon-i x}^{1+\varepsilon+i x} F_{1}(s ; y) \frac{(x+H)^{s}-x^{s}}{s} d s+O\left(x^{\varepsilon}\right) .
$$

Move the line of integration above to $\Re s=\sigma_{0}=\frac{24}{73}$. By the residue theorem, we have 


$$
\begin{aligned}
& \sum_{x<k \leq x+H} f_{1}(k ; y)=\operatorname{Res}_{s=1 / 2}+\operatorname{Res}_{s=1 / 3}+O\left(x^{\varepsilon}\right) \\
& \quad+\frac{1}{2 \pi i}\left(\int_{\sigma_{0}-i x}^{\sigma_{0}+i x}-\int_{1+\varepsilon+i x}^{\sigma_{0}+i x}-\int_{\sigma_{0}-i x}^{1+\varepsilon-i x}\right) F_{2}(s) F(s ; y) \frac{(x+H)^{s}-x^{s}}{s} d s
\end{aligned}
$$

where $\operatorname{Res}_{s=1 / 2,1 / 3}$ are the residues of $F_{2}(s) F(s ; y) \frac{(x+H)^{s}-x^{s}}{s}$ at $s=1 / 2,1 / 3$, respectively. By Lemma 4.4(i),

$$
\begin{aligned}
\operatorname{Res}_{s=1 / 2}+\operatorname{Res}_{s=1 / 3}= & c_{3}\left((x+H)^{1 / 2}-x^{1 / 2}\right) \sum_{n>y} \frac{\mu(d)}{d^{2}} \\
& -c_{4}\left((x+H)^{1 / 3}-x^{1 / 3}\right) \sum_{n>y} \frac{\mu(d)}{d^{4 / 3}} .
\end{aligned}
$$

By Lemma 4.4(ii) \& (iii), we easily get

$$
\frac{1}{2 \pi i}\left(\int_{1+\varepsilon-i x}^{\sigma_{0}-i x}+\int_{\sigma_{0}+i x}^{1+\varepsilon+i x}\right) \ll x^{\frac{127}{616}+\varepsilon} .
$$

Now we only need to estimate

$$
\begin{aligned}
\frac{1}{2 \pi i} \int_{\sigma_{0}-i x}^{\sigma_{0}+i x} & \ll\left|\int_{0}^{x} F_{2}\left(\sigma_{0}+i t\right) F\left(\sigma_{0}+i t ; y\right) \frac{(x+H)^{\sigma_{0}+i t}-x^{\sigma_{0}+i t}}{\sigma_{0}+i t} d t\right| \\
& \ll\left|\int_{0}^{y}\right|+\left|\int_{y}^{x}\right|,
\end{aligned}
$$

say. By Cauchy's equality, Lemma 4.4(iv) and Lemma 4.5, we have

$$
\begin{aligned}
\left|\int_{0}^{y}\right| & =\left|\int_{0}^{y} F_{2}\left(\sigma_{0}+i t\right) F\left(\sigma_{0}+i t ; y\right)\left(\int_{x}^{x+H} u^{\sigma_{0}-1+i t} d u\right) d t\right| \\
& \ll \int_{x}^{x+H} u^{\sigma_{0}-1} d u\left|\int_{0}^{y} F_{2}\left(\sigma_{0}+i t\right) F\left(\sigma_{0}+i t ; y\right) d t\right| \\
& \ll\left((x+H)^{\sigma_{0}}-x^{\sigma_{0}}\right)\left(\int_{0}^{y}\left|F_{2}\left(\sigma_{0}+i t\right)\right|^{2} d t\right)^{1 / 2}\left(\int_{0}^{y}\left|F\left(\sigma_{0}+i t ; y\right)\right|^{2} d t\right)^{1 / 2} \\
& \ll H x^{\sigma_{0}-1} y^{1 / 2}\left(\sum_{d>y}(y+d) d^{-8 \sigma_{0}}\right)^{1 / 2} \ll H x^{\sigma_{0}-1} y^{3 / 2-4 \sigma_{0}}
\end{aligned}
$$

and

$$
\begin{aligned}
\left|\int_{y}^{x}\right| & =\left|\int_{y}^{x} F_{2}\left(\sigma_{0}+i t\right) F\left(\sigma_{0}+i t ; y\right) \frac{(x+H)^{\sigma_{0}+i t}-x^{\sigma_{0}+i t}}{\sigma_{0}+i t} d t\right| \\
& \ll x^{\sigma_{0}} \max _{y \ll M \ll x} M^{-1}\left(\int_{M}^{2 M}\left|F_{2}\left(\sigma_{0}+i t\right)\right|^{2} d t\right)^{1 / 2}\left(\int_{M}^{2 M}\left|F\left(\sigma_{0}+i t ; y\right)\right|^{2} d t\right)^{1 / 2}
\end{aligned}
$$




$$
\begin{aligned}
& \ll x^{\sigma_{0}+\varepsilon} \max _{y \ll M \ll x} M^{-1 / 2}\left(\sum_{d>y}(M+d) d^{-8 \sigma_{0}}\right)^{1 / 2} \\
& \ll x^{\sigma_{0}+\varepsilon} \max _{y \ll M \ll x}\left(y^{1 / 2-4 \sigma_{0}}+M^{-1 / 2} y^{1-4 \sigma_{0}}\right) \ll x^{\sigma_{0}+\varepsilon} y^{1 / 2-4 \sigma_{0}} .
\end{aligned}
$$

Thus

$$
\frac{1}{2 \pi i} \int_{\sigma_{0}-i x}^{\sigma_{0}+i x} \ll H x^{\sigma_{0}-1} y^{3 / 2-4 \sigma_{0}}+x^{\sigma_{0}+\varepsilon} y^{1 / 2-4 \sigma_{0}} .
$$

Combining (4.6)-(4.9) with (4.5), we get

$$
\begin{aligned}
\sum_{d>y}= & c_{3}\left((x+H)^{1 / 2}-x^{1 / 2}\right) \sum_{n>y} \frac{\mu(d)}{d^{2}} \\
& -c_{4}\left((x+H)^{1 / 3}-x^{1 / 3}\right) \sum_{n>y} \frac{\mu(d)}{d^{4 / 3}} \\
& +O\left(x^{\frac{127}{616}+\varepsilon}+H x^{\sigma_{0}-1} y^{3 / 2-4 \sigma_{0}}+x^{\sigma_{0}+\varepsilon} y^{1 / 2-4 \sigma_{0}}\right) .
\end{aligned}
$$

This together with (4.3), (4.4) yields

$$
\begin{aligned}
L_{\text {area }}^{\prime}(x+H) & -L_{\text {area }}^{\prime}(x) \\
= & c_{3} \zeta(2)^{-1}\left((x+H)^{1 / 2}-x^{1 / 2}\right)-c_{4} \zeta(4 / 3)^{-1}\left((x+H)^{1 / 3}-x^{1 / 3}\right) \\
& +O\left(x^{\frac{127}{616}+\varepsilon}+x^{1 / 8+\varepsilon} y^{1 / 2}+H x^{\sigma_{0}-1} y^{3 / 2-4 \sigma_{0}}+x^{\sigma_{0}+\varepsilon} y^{1 / 2-4 \sigma_{0}}\right) \\
= & \frac{c_{3}}{2} \zeta(2)^{-1} H x^{-1 / 2}+O\left(x^{\frac{127}{616}+\varepsilon}\right)+O\left(x^{1 / 8+\varepsilon} y^{1 / 2}+x^{\sigma_{0}+\varepsilon} y^{1 / 2-4 \sigma_{0}}\right) \\
& +O\left(H x^{\sigma_{0}-1+\varepsilon} y^{3 / 2-4 \sigma_{0}}+H x^{-2 / 3}\right) .
\end{aligned}
$$

Take $y=x^{1 / 4-1 / 32 \sigma_{0}}$. On recalling $\sigma_{0}=\frac{24}{73}$, it is easy to check that the second $O$-term is $\ll x^{\frac{127}{616}+\varepsilon}$ and the third $O$-term is $\ll H x^{-1 / 2-\varepsilon}$. Thus

$$
\begin{aligned}
& L_{\text {area }}^{\prime}(x+H)-L_{\text {area }}^{\prime}(x) \\
& \quad=\frac{c_{3}}{2} \zeta(2)^{-1} H x^{-1 / 2}+O\left(H x^{-1 / 2-\varepsilon}+x^{\frac{127}{616}+\varepsilon}\right) .
\end{aligned}
$$

Now Theorem 1.3 follows from (4.11) and Lemma 4.1.

\section{References}

[1] R. C. Baker, The square-free divisor problem, Quart. J. Math. Oxford Ser. 45 (1994), 269-277.

[2] —, The square-free divisor problem II, ibid. 47 (1996), 133-146.

[3] J. Duttlinger und W. Schwarz, Über die Verteilung der Pythagoräischen Dreiecke, Colloq. Math. 43 (1980), 365-372.

[4] M. Filaseta and O. Trifonov, The distribution of fractional parts with applications to gap results in number theory, Proc. London Math. Soc. 73 (1996), 241-278. 
[5] E. Fouvry and H. Iwaniec, Exponential sums with monomials, J. Number Theory 33 (1989), 311-333.

[6] M. N. Huxley, Exponential sums and lattice points III, Proc. London Math. Soc. 87 (2003), 591-609.

[7] M. N. Huxley and W. G. Nowak, Primitive lattice points in convex planar domains, Acta Arith. 76 (1996), 271-283.

[8] J. Lambek and L. Moser, On the distribution of Pythagorean triangles, Pacific J. Math. 5 (1955), 73-83.

[9] D. H. Lehmer, A conjecture of Krishnaswami, Bull. Amer. Math. Soc. 54 (1948), 1185-1190.

[10] D. N. Lehmer, Asymptotic evaluation of certain totient sums, Amer. J. Math. 22 (1900), 293-335.

[11] H. Menzer, On the number of primitive Pythagorean triangles, Math. Nachr. 128 (1986), 129-133.

[12] S. H. Min, The Methods of Number Theory, Science Press, Beijing, 1981 (in Chinese).

[13] H. L. Montgomery and R. C. Vaughan, The distribution of squarefree numbers, in: Recent Progress in Analytic Number Theory (Durham, 1979), Vol. 1, Academic Press, London, 1981, 247-256.

[14] W. Müller and W. G. Nowak, Lattice points in planar domains: applications of Huxley's "discrete Hardy-Littlewood method", in: Number-Theoretic Analysis (Vienna, 1988-1989), Lecture Notes in Math. 1452, Springer, 1990, 139-164.

[15] W. Müller, W. G. Nowak and H. Menzer, On the number of primitive Pythagorean triangles, Ann. Sci. Math. Québec 12 (1988), 263-273.

[16] W. G. Nowak, Primitive lattice points in starlike planar sets, Pacific J. Math. 170 (1997), 163-178.

[17] C. D. Pan and C. B. Pan, Foundations of the Analytic Number Theory, Science Press, Beijing, 1991 (in Chinese).

[18] O. Robert and P. Sargos, Three-dimensional exponential sums with monomials, J. Reine Angew. Math. 591 (2006), 1-20.

[19] J. D. Vaaler, Some extremal problems in Fourier analysis, Bull. Amer. Math. Soc. 12 (1985), 183-216.

[20] I. M. Vinogradov, Special Variants of the Method of Trigonometric Sums, Nauka, Moscow, 1976 (in Russian); English transl. in Selected Works, Springer, 1985.

[21] R. E. Wild, On the number of primitive triangles with area less than $n$, Pacific J. Math. 5 (1955), 85-91.

[22] W. G. Zhai, On the number of primitive Pythagorean triangles, Acta Arith. 105 (2002), 387-403.

[23] —, On primitive lattice points in planar domains, ibid. 109 (2003), 1-26.

Kui Liu

Department of Mathematics

Shandong University

Jinan, Shandong 250100, P.R. China

E-mail: liukui84@sdu.edu.cn 